\title{
Puntura a Occhiello: cosa c'è di nuovo?
}

\author{
Marisa Pegoraro
}

\author{
EDTNA/ERCA Filiale Italiana
}

THE BUTTONHOLE TECHNIQUE: WHAT'S NEW?

Abstract: The buttonhole technique (BH) was introduced in Italy in 2005 after data obtained by foreign centers in the EDTNA/ERCA setting provided evidence of its advantages. Its correct application requires advanced clinical expertise within the nursing team. An example is the vascular access nurse, who teaches, monitors, and updates clinical protocols. International data have shown a correlation between nurses' adherence to protocol, their clinical practice skills, and BH outcomes. Shortcuts in the application of BH could lead to complications, the worst of which are access infections. Professional motivation and proper training of the nursing team play a pivotal role in activating the quality improvement loop. Proposing and supporting new clinical practice and organization models is part of the mission of any professional society.

Key words: AVF, Cannulation, Nursing roles, Advanced clinical practice

Conflict of interest: None.

Ricevuto: 23 Aprile 2013; Accettato: 8 Maggio 2013

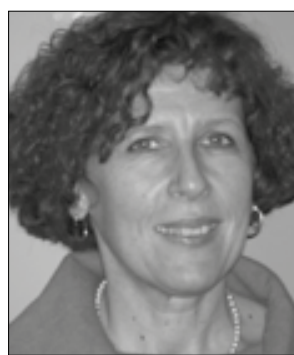

Marisa Pegoraro
La campagna divulgativa sull'utilizzo della tecnica della puntura a occhiello (o buttonhole) della fistola artero-venosa (FAV) nativa è iniziata nel 2005, dopo che ebbi modo di ascoltare la presentazione di Tony Goovaerts, di Bruxelles, a cui fece seguito una visita nel suo reparto. Decisiva è stata la vista delle braccia dei "suoi" pazienti: erano molto migliori di quelle dei "miei", anche se io ritenevo di essermene sempre presa buona cura! Decidere di attivare una campagna divulgativa ritengo sia stata veramente una "buona idea"!! Lo dico con gioia, pensando ai pazienti che ne hanno beneficiato, e con un certo orgoglio, pensando a tutti i colleghi che si sono sentiti a loro volta portatori di novità e, si spera, di benefici. Ho dato retta al mio istinto personale e professionale, ho potuto fare tesoro dei vantaggi di essere socia di un grande gruppo professionale (l'EDTNA/ERCA) e di avere avuto il privilegio di poter condividere dati e opinioni con dei colleghi più esperti... Una di quelle serendipiche condizioni in cui, da poco, viene fuori molto. Chi pratica la puntura a occhiello e raccoglie dati da tempo ha scritto: "il buttonhole non è solo una tecnica diversa, ma, piuttosto, un diverso sistema di approccio e gestione della FAV nativa" (1). Questo sta a significare che, per dare esiti positivi ed efficaci, la tecnica necessita di essere inserita in uno schema organizzativo, come, dove, per quanto, e ... di qualcuno che tenga le "redini", supervisionando il lavoro e verificandone gli esiti. Per usare termini che rispecchiano meglio la tendenza professionale attuale, la tecnica richiede la presenza di figure infermieristiche di "competenza avanzata", cioè di qualcuno che, per impegno, ingegno o contratto, ha la responsabilità di applicare la tecnica, di insegnarla agli altri, di controllare che sia applicata correttamente e, infine, di verificarne gli esiti clinici nel medio e nel lungo termine. Nel contesto internazionale è noto il ruolo della "Vascular Access Nurse" (2, 3). Vi potrebbe attraversare il pensiero che "...allora è troppo complicato applicarla, meglio il solito buco veloce e senza inghippi”. Come risposta, riprendo le parole di un collega che da lunga data applica la tecnica nel suo centro ospedaliero, Marc Boogaerts di Lione (4): “.... credo gli infermieri abbiano il dovere etico di scegliere la metodica di puntura che induce meno dolore, meno deturpazioni e meno errori'. Appena lessi, alcuni anni fa, la frase, mi parve un po' eccessiva, mentre oggi concordo a pienamente. Effettivamente, le differenze, per i nostri pazienti, non sono solo meno dolore, un braccio quasi normale e meno stress da "buco mancato", ... no, la differenza è... "sentirsi meno malati, meno brutti e meno fragili". Insomma: una migliore qualità di vita.

Alcuni centri, confrontando i dati dei pazienti in cui viene usata la tecnica a occhiello versus quelli in tecnica ad area/rotazione, hanno anche notato un minore numero di interventi diagnostico-correttivi (angioplastiche, revisioni chirurgiche...) a favore del gruppo a occhiello $(1,5)$. È un interessante dato di tendenza nell'economia dei servizi pubblici: una migliore qualità ha un costo minore!! Per noi infermieri, in questo caso, le migliori economie sono un'espressione di professionalità. Applicare la tecnica nel contesto e nel modo corretti esalta la necessità di un dialogo "professionale" con i colleghi, con il paziente e con gli altri professionisti, esalta la responsabilizzazione e la conseguente acquisizione di competenze ed esalta la necessità di raccogliere dati per fare confronti (quello che gli anglosassoni chiamano "benchmarking"), per giustificare l'acquisto degli aghi, il tempo dedicato a insegnare l'autopuntura, i minuti dedicati alla rimozione della crosta e così via. Però, solo facendo così saremo certi di fare bene, perché l'occhiello... è insidioso; se non è contestualizzato, se il 
protocollo non viene seguito da tutti, se in molti pensano di poter fare di testa propria, allora l'occhiello potrebbe fare danni. Danni che non si vedono subito, ma a distanza di tempo e, a volte, anche di spazio: parlo dell'infezione locale e di quella metastatica. Perciò, tanto sono convinta che sia una tecnica valida quanto sono sicura che più il contesto è complesso (centro ospedaliero, tanti pazienti, tanti infermieri, ecc.) più è necessaria un'organizzazione delle competenze e delle pratiche (6). Sfatiamo il concetto che tutti debbano fare tutto, sempre. No, la professionalità richiede progressività: una fisiologica curva di apprendimento, a cui tutti possono accedere in progressione, che potrà essere accelerata dalla motivazione, dall'abilità e, in parte, anche dalla necessità, ma mai sottostimata, mai svalutata, mai obbligata. L'occhiello fatto con disaffezione, superficialità e opportunismo ha una grossa probabilità di essere dannoso. Ovviamente è più facile dare la colpa oggettiva a una tecnica, piuttosto che rilevare delle responsabilità soggettive degli operatori.

Abbiamo avuto modo di comprendere, dopo i primi anni di utilizzo dei cateteri venosi centrali (CVC) permanenti, che non sono i cateteri che inducono le infezioni, ma piuttosto come i cateteri vengono impiantati, maneggiati e gestiti e che l'uso proprio dell'organo cerebrale non è precluso a priori agli infermieri, se non per sola scelta personale. Oggi consideriamo il CVC una necessità come accesso vascolare "ultimo" (o primo). Allo stesso modo, dovremmo considerare la puntura a occhiello una necessità per preservare nel tempo la funzione della FAV e per sostenere la migliore qualità di vita dei nostri pazienti.

Ho recentemente svolto una revisione bibliografica che ha evidenziato anche nel contesto internazionale dati a macchia di leopardo: centri con esiti brillanti e centri con esiti scarsi ed esiti modesti all'inizio, poi in crescendo, se le infermiere sono motivate, e in calando, se il contesto demotiva o se si allenta l'attenzione. Quali siano le componenti della "motivazione" è stato oggetto di interessanti studi socio-antropologici di carattere qualitativo che meritano un approfondimento specifico. Alcuni ospedali negli USA hanno strutturato le componenti della motivazione in elementi di "magnetismo" utilizzati per ritenere e attirare personale qualificato (7). Il magnetismo professionale trova ragioni primarie nella soddisfazione di vedere un beneficio, di imparare qualcosa di nuovo, di poter condividere, di progredire nella competenza (conoscenza e abilità) e con queste cose, poi, arrivano anche le incentivazioni stipendiali di progetto, di innovazione e di ruolo (8). Certo, non tutte queste componenti dipendono da noi, ma noi infermieri dobbiamo fare la nostra parte. Lo scenario del mondo del lavoro sarà sempre più volto a correlare $\mathrm{i}$ compensi con gli esiti. Giustificare quanto il nostro lavoro concorra al raggiungimento e al mantenimento dei buoni esiti è responsabilità di ogni singolo professionista. Definire quali siano le buone pratiche è una delle "mission" delle società scientifiche, una tra queste l'EDTNA/ERCA, e su questo ci stiamo muovendo. Mi auguro che molti ancora siano interessati a concorrere al benessere comune.

\section{Riassunto}

La divulgazione della Puntura ad Occhiello - o Buttonhole (BH) - in Italia iniziò nel 2005 sostenuta da dati provenienti da alcuni centri esteri, confluenti nel circuito dell'EDTNA/ERCA. La corretta applicazione del $\mathrm{BH}$ esalta la necessità di figure di competenza clinica avanzata nel gruppo infermieristico come la figura dell'Infermiera degli Accessi Vascolari con competenze di formazione, monitoraggio e aggiornamento dei protocolli clinici. I dati internazionali mostrano una correlazione tra l'aderenza ai protocolli clinici, la competenza nella pratica professionale ed i risultati della puntura ad occhiello. Le vie brevi e veloci nell'applicazione del BH possono indurre ad avversi esiti clinici, i peggiori delle quali sono le infezioni dell'accesso. La motivazione professionale e la corretta formazione del gruppo infermieristico giocano ruoli chiave nell'attivazione di programmi di miglioramento qualitativo. Proporre e sostenere nuove pratiche cliniche e modelli organizzativi è parte del mandato di ogni organizzazione professionale.

Parole Chiave: Incannulazione di FAV, Ruoli infermieristici, Pratiche cliniche avanzate

Dichiarazione di conflitto di interessi: L'Autore dichiara di non avere conflitto di interessi

Indirizzo degli Autori:

Dr.ssa Marisa Pegoraro

EDTNA/ERCA Filiale Italiana

c/o DIALISI CAL Corsico

Via Travaglia 5

20094 Corsico

marisa pegoraro@fastwebnet.it

\section{Bibliografia}

1. van Loon MM, Goovaerts T, Kessels AG, van der Sande FM, Tordoir JH. Buttonhole needling of haemodialysis arteriovenous fistulae results in less complications and interventions compared to the ropeladder technique. Nephrol Dial Transplant 2010; 25 (1): 225-30.

2. Waterhouse D. Vascular access: a role for a renal nurse clinician. EDTNA ERCA J 2002; 28 (2): 64-6, 69.

3. Polkinghorne KR, Seneviratne M, Kerr PG. Effect of a vascular access nurse coordinator to reduce central venous catheter use in incident hemodialysis patients: a quality improvement report. Am J Kidney Dis 2009; 53 (1): 99-106.

4. Murcutt G. Buttonhole cannulation: should this become the default technique for dialysis patients with native fistulas? Summary of the EDTNA/ERCA Journal Club discussion Autumn 2007. J Ren Care 2008; 34 (2): 101-8.

5. Pergolotti A, Rich E, Lock K. The effect of the buttonhole method vs. the traditional method of AV fistula cannulation on hemostasis, needle stick pain, pre-needle stick anxiety, and presence of aneurysms in ambulatory patients on hemodialysis. Nephrol Nurs J 2011; 38 (4): 333-6.

6. Labriola L, Jadoul M. Infectious complications following conversion to buttonhole cannulation. Clin Nephrol 2011; 76 (5): 423.

7. Pinkerton S. The Magnet view: pursuing ANCC Magnet recognition as a system or individual organization. Nurs Econ 2008; 26 (5): 323-4.

8. Shaver KH, Lacey LM. Job and career satisfaction among staff nurses: effects of job setting and environment. J Nurs Adm 2003; 33 (3): 166-72. 